Original Paper http://ajol.info/index.php/ijbcs http://indexmedicus.afro.who.int

\title{
Assessing tree effect on total soil carbon in agroforestry parklands systems along a rainfall gradient in Burkina Faso (West Africa)
}

\author{
Yacouba Noël COULIBALY ${ }^{*}$, Jules BAYALA ${ }^{2}$, Thomas GAISER ${ }^{3}$ and \\ Gérard ZOMBRE ${ }^{4}$
}
${ }^{1}$ Institut de l'Environnement et de Recherches Agricoles (INERA), Station de Recherches Environnementales et Agricoles de Farako-Bâ, 01 BP 910, Bobo-Dioulasso, Burkina Faso.
${ }^{2}$ World Agroforestry (ICRAF), ICRAF-WCA/ Sahel Node, BP E5118 Bamako, Mali.
${ }^{3}$ University of Bonn, Institute of Crop Science and Resource Conservation, Katzenburgweg 5 D-53115 Bonn.
${ }^{4}$ Université Joseph Ki-Zerbo, Ecole Doctorale Sciences et Techniques, 03 BP 7021, Ouagadougou 03, Burkina Faso.
${ }^{*}$ Corresponding author; E-mail : yacoubacoulibaly2002@yahoo.fr ; Tel. (00226) 78873475

\begin{tabular}{lll}
\hline Received: 30-07-2020 & Accepted: 18-12-2020 & Published: 31-12-2020 \\
\hline
\end{tabular}

\begin{abstract}
Trees contribution in improving soil carbon is well established, but few works addressed how this was affected by a climatic gradient. This research investigated effects of Vitellaria paradoxa C. F Gaertn and Parkia biglobosa (Jacq.) Benth on total soil carbon in parklands along a rainfall gradient for recommendations of tree species which better improve soil carbon under specific climatic conditions for parklands adaptation to climate change. Total soil carbon at topsoil and subsoil layers measured using spectrophotometry infrared method, was higher when rainfall increased and were respectively $(1.598 \pm 0.040 ; 1.033 \pm 0.022 ; 0.834 \pm$ $0.014 ; 0.857 \pm 0.016 \%)$. It was higher at topsoil $(0.529 \pm 0.015 \%)$ and subsoil $(0.282 \pm 0.019 \%)$ under $V$. paradoxa when rainfall decreased while it was higher under $P$. biglobosa and $V$. paradoxa when rainfall increased slightly. Its improvement was higher under $V$. paradoxa and $P$. biglobosa when rainfall respectively decreased and increased. A decrease trend of total soil carbon under both tree species from trunk to outside the canopy whatever rainfall levels and soil layers was observed. Tree species choice could play an important role in improving total soil carbon and crop productivity according to rainfall level for parklands adaptation to climate change.
\end{abstract}

(C) 2020 International Formulae Group. All rights reserved.

Keywords: Management options, topsoil, precipitation, subsoil, climate.

\section{INTRODUCTION}

The soils in Sahelian region are characterized by a negative mineral balance due to the insufficient use of organic materials and low use of fertilizers to compensate for exported nutrients by the crops (Bationo et al., 2007). Agroforestry practices have the potential in improving soil carbon through environmental services provided by trees on farms (Ndiaye et al., 2012; Abdou et al., 2013; Aliou et al., 2013; Bayala et al., 2019). Ndiaye et al. (2012) reported a positive impact on crop productivity when soil carbon has improved through the practice of agroforestry. Most of the studies related to the impact of trees in improving soil carbon in agroforestry 
parklands in the Sahel region focused on one tree species and on one site (Traore et al., 2004) while the positive effect of tree on soil fertility depends on several factors such as tree species, climate, soil characteristics, altitude and land use (Lorenz et al., 2014; Mathayo et al., 2016; Sileshi, 2016; Bayala et al., 2019). Furthermore, the magnitude of the impact of tree species on soil organic carbon improvement according to a rainfall gradient is not well documented according to our literature review (Lorenz et al., 2014). This research aimed at investigating the effects of Vitellaria paradoxa C. F Gaertn and Parkia biglobosa (Jacq.) Benth on total soil carbon in agroforestry parklands along a rainfall gradient for recommendations of tree species which better improve soil carbon under specific climatic conditions for parklands adaptation to climate change.

\section{MATERIALS AND METHODS \\ Site description}

Soil survey was conducted at three different sites along an increasing rainfall gradient: Tougouri (with the lowest rainfall level studied) located at $13^{\circ} 18^{\prime} 59^{\prime \prime}$ latitude North and $-3^{\circ} 12^{\prime} 1 "$ longitude West in the Sahelian zone (northern part). Tougouri belongs to the administrative region centralnorth and the district of Namentenga; Nobere (with the middle rainfall level studied) located at $11^{\circ} 33^{\prime} 29^{\prime \prime}$ latitude North and $-1^{\circ} 12^{\prime} 16^{\prime \prime}$ longitude West in the Sudano-Sahelian savanna (central part). Nobere belongs to the administrative region central south and the district of Zoundweogo. Sokouraba located at $10^{\circ} 51^{\prime} 00^{\prime \prime}$ latitude North and $-5^{\circ} 11^{\prime} 00^{\prime \prime}$ longitude West in the Sudano-Guinean savanna (southern part). Sokouraba (with the highest rainfall level studied) belongs to the administrative region Haut-Bassins and the district of Kenedougou. The soils of the three sites are generally poor and have low N, MO and $\mathrm{P}$ contents. In addition, they are weakly acidic with low CEC (Table 1). Average rainfall and temperature (year 1980-2013) were $557 \mathrm{~mm}$ and $26.6{ }^{\circ} \mathrm{C}$ in Tougouri respectively, $859 \mathrm{~mm}$ and $25.7{ }^{\circ} \mathrm{C}$ in Nobere, and $1061 \mathrm{~mm}$ and $25.1{ }^{\circ} \mathrm{C}$ in Sokouraba (DGM, 2013). The average rainfall totaled 620, 775 and $927 \mathrm{~mm}$, respectively in
Tougouri, Nobere and Sokouraba during the two years (2011 and 2012) of measurements.

The secondary data from the report of

the National Institute of Statistic and Demography of Burkina Faso (INSD, 2018) related to population growth and population density (1985-2006) and livestock development (2011-2014) of the administrative region or district from where the study site was located, was used for the socio-economic characterization. For the appreciation of livestock development, the total number of heads for each year including cattle, sheep and goats was considered. The analysis of the secondary data related to population growth showed an increase of population in all the districts to which belong the different study sites with the highest increase at Tougouri over the period 19852006 (Figure 1A). The population density followed the same trend of increase in all the districts to which belong the three study sites with the highest population density observed at Tougouri and Nobere over the period 19852006 (Figure 1B). The analysis of the secondary data related to the livestock sector over the period 2011-2014 showed an increase of the number of heads in districts to which belong Tougouri and Nobere (Figure 2). But in district to which belongs Sokouraba, a decline of the number of heads of livestock over the time period studied was observed (Figure 2). Inversely, from 2012 an increase trend of the number of heads of livestock was observed in districts to which belong Tougouri and Nobere leading to the highest number of heads of livestock in 2014 for these two later sites (Figure 2).

\section{Experimental design}

The studied parklands systems consisted of a parkland system with two native tree species: $V$. paradoxa and $P$. biglobosa. The area around each of the sampled trees was split into three concentric tree influence zones and a control plot which were:

- Zone A - from tree trunk to half of the crown radius of the tree;

- Zone B - from half of the crown radius of the tree up to the edge of the crown; 
- Zone C - from the edge of the tree crown up to $3 \mathrm{~m}$ away; and

- Zone $\mathrm{H}$ - a control plot for crop in monoculture which was an area of 4 x $4 \mathrm{~m}$ situated at least $40 \mathrm{~m}$ away from the edge of the crown and unshaded by any of the surrounding trees at any time of the day throughout the cropping season.

This design was replicated eight times for each tree species at each site to give a total of sixty-four (= 8 reps $\times 2$ species $\times 4$ zones) sampling positions in Sokouraba, Nobere and Tougouri.

\section{Data collection}

Soil sampling was conducted randomly at two points in each of the concentric zones and control plot at $10 \mathrm{~cm}$ interval depth up to $40 \mathrm{~cm}(0-10 ; 10-20 ; 20-30$ and $30-40 \mathrm{~cm})$ using an auger of $5 \mathrm{~cm}$ diameter with a volume of $250 \mathrm{~cm}^{3}$. The two soil samples for the same depth and zone were mixed to have a composite sample of $500 \mathrm{~cm}^{3}$ for soil analysis. For this study, the topsoil was layers $0-10$ $\mathrm{cm}$ and $10-20 \mathrm{~cm}$ and the subsoil were layers $20-30 \mathrm{~cm}$ and $30-40 \mathrm{~cm}$. The spectrophotometry infrared method was used for the total soil carbon measurements (Shepherd and Walsh, 2002; Du and Zhou, 2009). The principle of this method is based on the fact that different soil components absorb the near rays differently according to their level of importance in the sample. The composites soil samples were dried at open air, sieved using a sieve of $2 \mathrm{~mm}$ mesh and scanned at Near Infrared (NIR) and middle infrared (MIR) using the spectrophotometer «Bruker Fourier-Transform MultiPurpose
Analyzer spectrometers (MPA)» (Bruker Optik GmbH, Germany) equipped with a software which predicts the total soil carbon content. For the purpose of scanning, soil subsamples of about $20 \mathrm{~g}$ from each composite dried soil samples were taken and put into a petri dish. Moreover, $10 \%$ of the whole soil samples were selected from which total soil carbon was analyzed using a humid chemistry in laboratory. The results from the humid chemistry in laboratory were used to estimate the accuracy of the results predicted by the software integrated in the spectrophotometer through calibration.

The quantitative parameter used to assess the magnitude of tree species effects on total soil carbon at the different study site was the difference between the average value of total soil carbon in zones under trees with the average value in control plot. The higher the difference, the stronger should be the magnitude.

\section{Statistical analysis}

The effect of rainfall gradient (sites), tree species and their interaction on total soil carbon at topsoil and subsoil layers were tested using the general model of ANOVA. When the differences among the means were significant with ANOVA, they were separated by the test of Student-Newman Keuils at $5 \%$. The pairwise t-tests were used to analyze the differences of total soil carbon between zones associated to the tree species at each of the study sites for topsoil and subsoil layers. The analyses have been done using the software XLSTAT 2018.

Table 1: Soil characteristics in the three study sites Tougouri (with the lowest rainfall level studied), Nobere (with the middle rainfall level studied) and Sokouraba (with the highest rainfall level studied) in Burkina Faso (West Africa). The values are the average of top $50 \mathrm{~cm}$ soil layer.

\begin{tabular}{lccc}
\hline Parameters & Tougouri & Nobere & Sokouraba \\
\hline$\%$ clay & 42,6 & 33,8 & 56,1 \\
\hline$\%$ Silt & 25 & 25,6 & 23,3 \\
\hline$\%$ Sand & 32,4 & 40,6 & 20,6 \\
\hline CEC (meq/100 g) & 10,13 & 5,81 & 9,34 \\
\hline Organic matter $(\%)$ & 0,43 & 0,39 & 1,05 \\
\hline N content $(\%)$ & 0,03 & 0,02 & 0,07 \\
\hline P content (P-Bray) $(\mathrm{ppm})$ & 2,2 & 9,56 & 5,38 \\
\hline $\mathrm{pH}$ & 5,92 & 6,43 & 5,71 \\
\hline
\end{tabular}



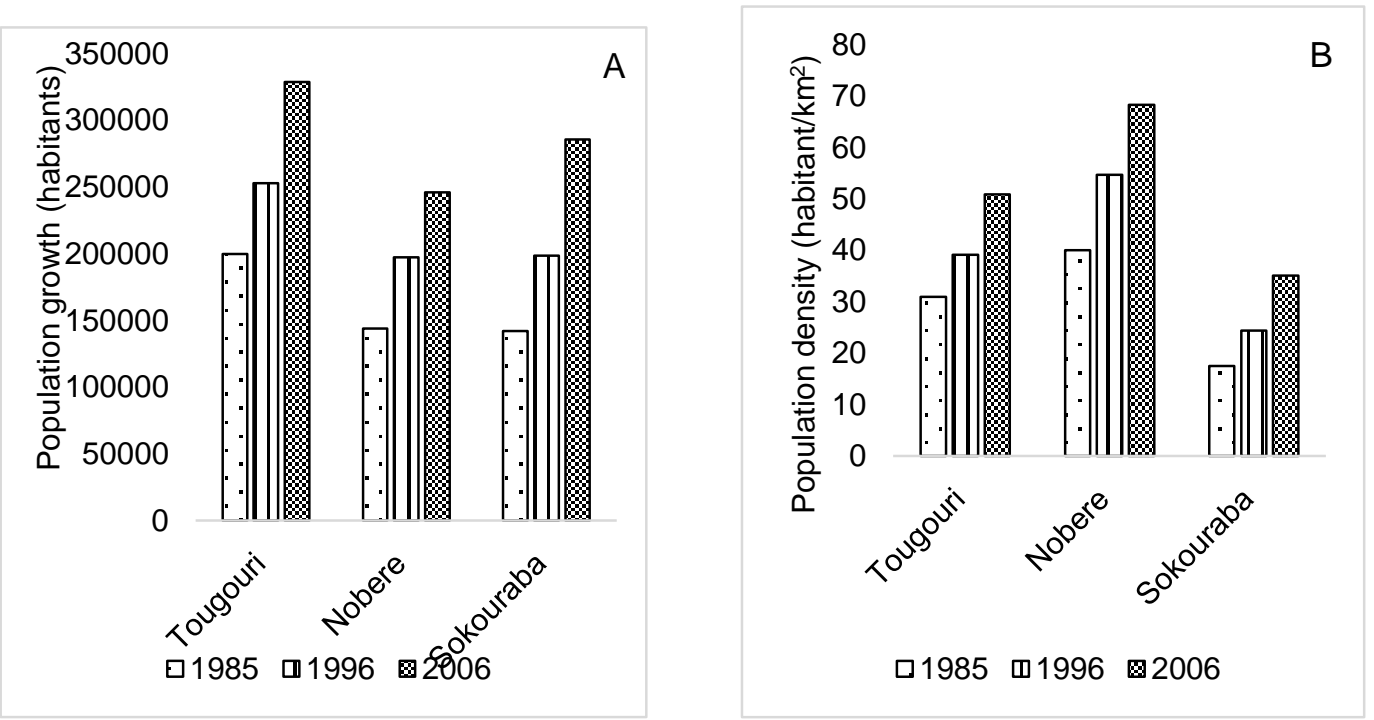

Figure 1: Population growth (A) and population density (B) variation over the time period 19852006 in the districts to which belongs the different study sites.

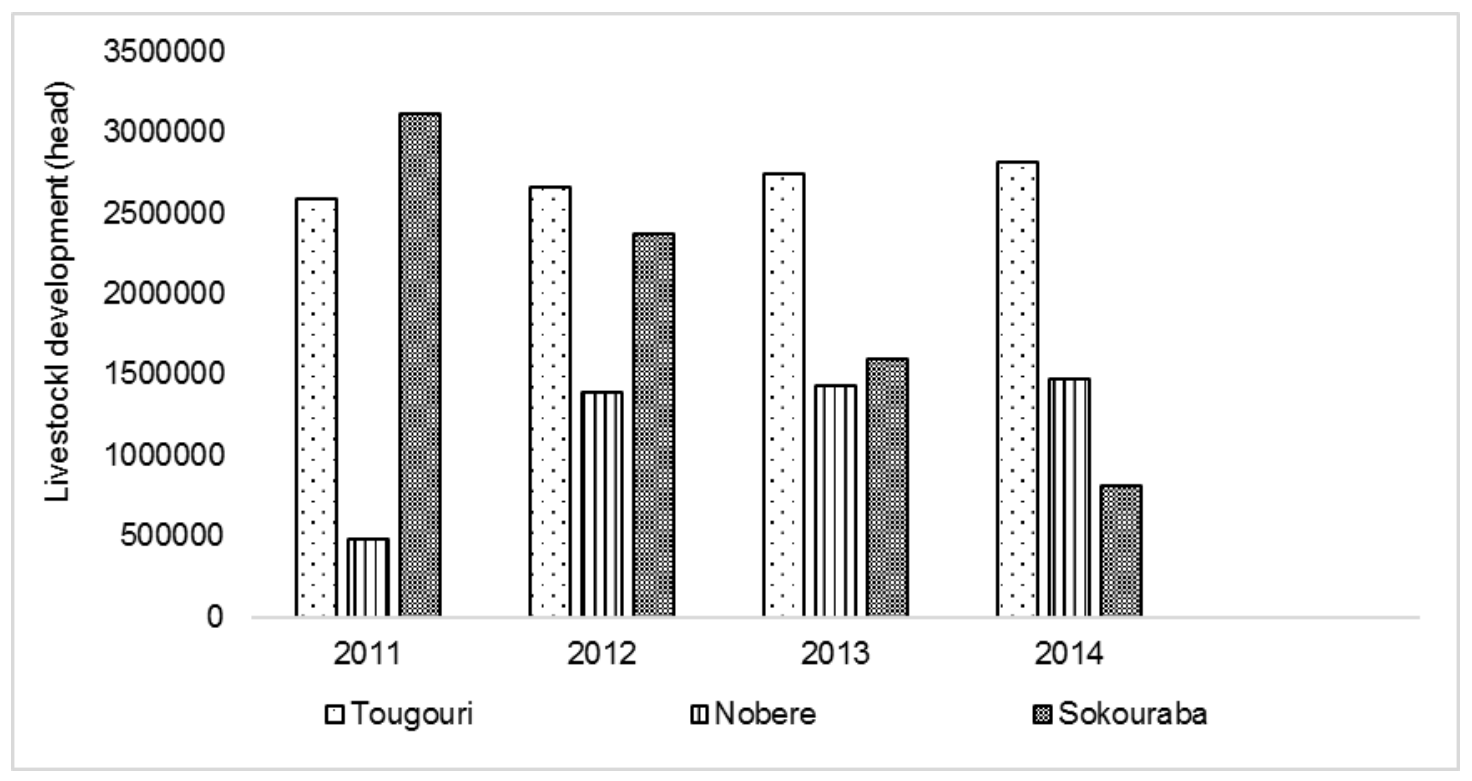

Figure 2 : Livestock development over the time period 2011-2014 in the districts to which belongs the different study sites.

\section{RESULTS}

The results of ANOVA showed highly significant difference of total soil carbon according to rainfall gradient (sites) for the topsoil and subsoil layers (Table 2). Total soil carbon increased with rainfall increase at topsoil and subsoil layers (Figure 3).
The results of ANOVA did not show significant difference of total soil carbon between tree species for topsoil and subsoil layers (Table 2).

The results of ANOVA showed significant interaction between rainfall gradient (sites) and tree species of total soil 
carbon at topsoil layer $10-20 \mathrm{~cm}$ (Table 2). Total soil carbon was not significantly different between both tree species at the highest rainfall level (Sokouraba) while it was higher at the lowest rainfall level (Tougouri) under $V$. paradoxa at the topsoil layer 10-20 $\mathrm{cm}$ (Figure 4). The total soil carbon was significantly higher under $P$. biglobosa at middle rainfall level (Nobere) at the topsoil layer 10-20 cm (Figure 4). At topsoil layer 10$20 \mathrm{~cm}$, total soil carbon under both $P$. biglobosa and $V$. paradoxa was higher at the highest rainfall level (Figure 4).

The results of ANOVA showed significant interaction between rainfall gradient (sites) and tree species of total soil carbon at subsoil layer $30-40 \mathrm{~cm}$ (Table 2). The total soil carbon at the subsoil layer 30-40 $\mathrm{cm}$ was not significantly different between both tree species at the highest and middle rainfall levels while it was higher under $V$. paradoxa.at the lowest rainfall level (Figure 5). At the subsoil layer $30-40 \mathrm{~cm}$, total soil carbon under both $P$. biglobosa and $V$. paradoxa was higher at the highest rainfall level (Figure 5).

The results of the pairwise t-tests showed some significant differences of total soil carbon between zones for a reduced number of pairs for $V$. paradoxa as well as for $P$. biglobosa at the highest, middle and lowest rainfall levels at topsoil and subsoil layers. The composite nature of the samples and the accuracy of spectrometry method are factors to be considered for lack of more significant difference of pairs.

At the lowest rainfall level, the total soil carbon was significantly different between zones A-B at the topsoil layer 10-20 $\mathrm{cm}$ and between zones A-C and A-H at the subsoil layers under V. paradoxa (Table 3). At the topsoil layer $10-20 \mathrm{~cm}$ and subsoil layers 20- $30 \mathrm{~cm}, 30-40 \mathrm{~cm}$ at the lowest rainfall level, total soil carbon was significantly higher in zone A under V. paradoxa (Figure 6A). At the lowest rainfall level, the total soil carbon was significantly different between zones A-C, B-H and $\mathrm{C}-\mathrm{H}$ at the topsoil layer
10-20 $\mathrm{cm}$ under $P$. biglobosa (Table 3). The total soil carbon was significantly higher in zone A under P. biglobosa (Figure 6B).

At the middle rainfall level, the total soil carbon was significantly different between zones A-C at the topsoil layer 10-20 cm under $V$. paradoxa (Table 4) with higher total soil carbon in zone A (Figure 7A). At the middle rainfall level, the total soil carbon was significantly different between zones A-B, A$\mathrm{C}$ and B-C under P. biglobosa at the topsoil layers $0-10 \mathrm{~cm}$ and $10-20 \mathrm{~cm}$ (Table 4) with higher total soil carbon in zone A (Figure 7B).

At the highest rainfall level, the total soil carbon was significantly different between zones A-C and A-H under $V$. paradoxa at topsoil layers $0-10 \mathrm{~cm}$ and $10-20$ $\mathrm{cm}$ (Table 5) with higher total soil carbon in zone A (Figure 8A). At the highest rainfall level, the total soil carbon was significantly different between zones A-C and B-H at the subsoil layer $30-40 \mathrm{~cm}$ (Table 5) under $V$. paradoxa with higher total soil carbon in zone A (Figure 8A). At the highest rainfall level, the total soil carbon was significantly different between zones A-B, A-C, A-H and B-H at the topsoil and subsoil layers (Table 5) under $P$. biglobosa with higher total soil carbon in zone A (Figure 8B).

In general, the pairwise t-tests showed a decrease trend of total soil carbon from zone A under both tree species, at the topsoil and subsoil layers for all the rainfall levels. Moreover, in general, higher total soil carbon at topsoil layers compared to subsoil layers in zones under trees for all sites under both tree species were observed

The results about the magnitude of trees species effect on total soil carbon was positive globally and showed that under $V$. paradoxa as well as $P$. biglobosa, the highest magnitude was observed at Sokouraba with the highest rainfall level (Table 6). At Tougouri with the lowest rainfall level, the magnitude was higher under $V$. paradoxa while at Nobere with the middle rainfall level and Sokouraba with the highest rainfall level, it was higher under $P$. biglobosa (Table 6). 
Table 2: ANOVA results for rainfall gradient (sites), tree species and their interactions effect on total soil carbon at topsoil and subsoil layers in agroforestry parklands of Burkina Faso (West Africa).

\begin{tabular}{|c|c|c|c|c|c|c|}
\hline Soil layers $(\mathbf{c m})$ & Source & DDL & Sum of squares & Mean of squares & $\mathbf{F}$ & $\operatorname{Pr}>F$ \\
\hline \multirow{3}{*}{010} & Site & 2 & 53,248 & 26,624 & 367,353 & $<0,0001$ \\
\hline & Species & 1 & 0,074 & 0,074 & 1,019 & 0,356 \\
\hline & Site*Species & 2 & 0,303 & 0,151 & 2,088 & 0,159 \\
\hline \multirow{3}{*}{1020} & Site & 2 & 14,539 & 7,269 & 350,704 & $<0,0001$ \\
\hline & Species & 1 & 0,073 & 0,073 & 3,523 & 0,09 \\
\hline & Site*Species & 2 & 0,191 & 0,095 & 4,607 & 0,024 \\
\hline \multirow{3}{*}{2030} & Site & 2 & 7,013 & 3,507 & 188,927 & $<0,0001$ \\
\hline & Species & 1 & 0,027 & 0,027 & 1,437 & 0,246 \\
\hline & Site*Species & 2 & 0,055 & 0,027 & 1,481 & 0,25 \\
\hline \multirow{3}{*}{3040} & Site & 2 & 14,642 & 7,326 & 416,097 & $<0,0001$ \\
\hline & Species & 1 & 0,019 & 0,019 & 1,107 & 0,294 \\
\hline & Site*Species & 2 & 0,122 & 0,061 & 3,457 & 0.034 \\
\hline
\end{tabular}

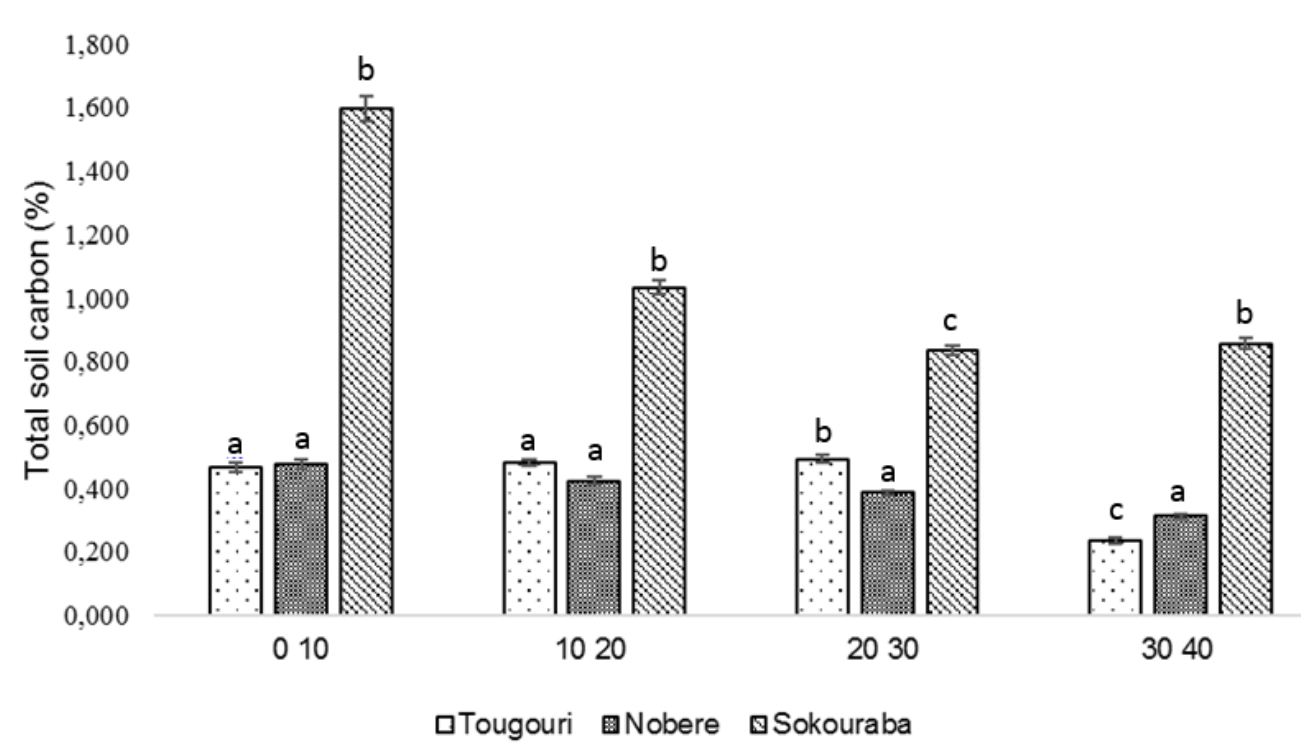

Figure 3: Total soil carbon variation according to rainfall gradient (sites) at topsoil and subsoil layers in agroforestry parklands systems in Burkina Faso (West Africa). 


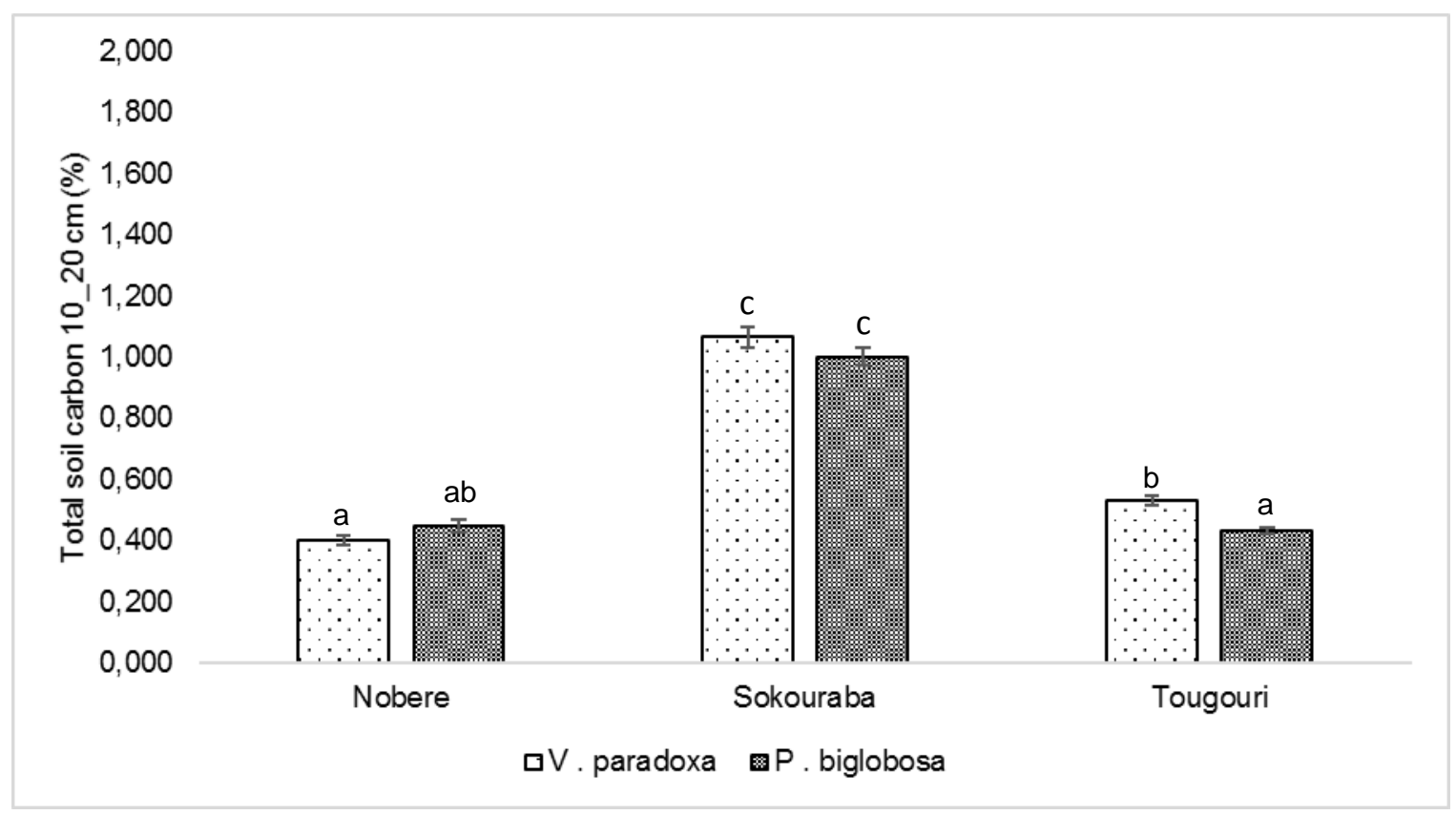

Figure 4: Total soil carbon variation under $V$. paradoxa and P. biglobosa according to the rainfall gradient (sites) at the topsoil layer 10-20 cm in agroforestry parklands systems in Burkina Faso (West Africa).

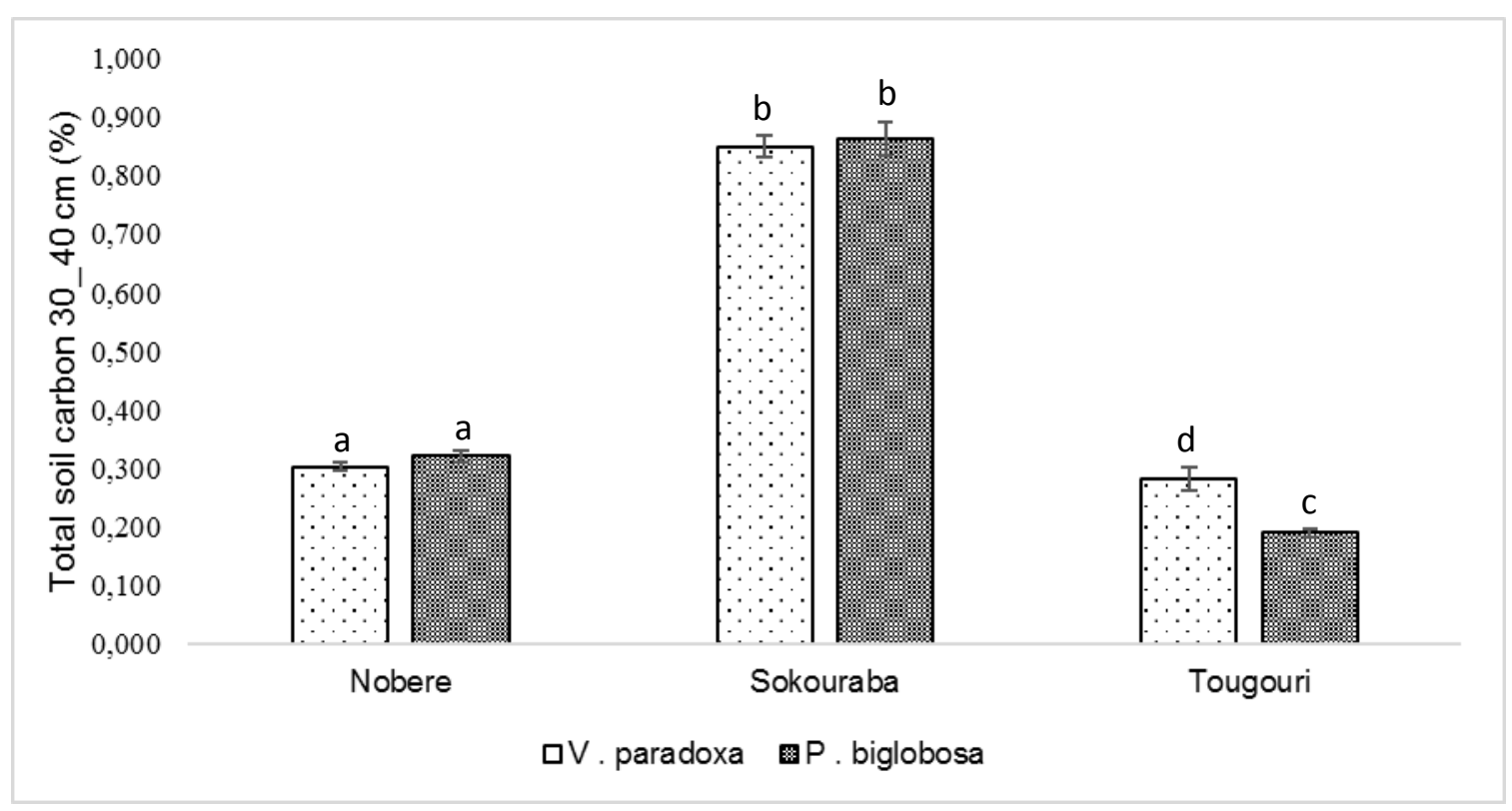

Figure 5: Total soil carbon variation under $V$. paradoxa and $P$. biglobosa according to the rainfall gradient (sites) at the subsoil layer $30-40 \mathrm{~cm}$ in agroforestry parklands systems in Burkina Faso (West Africa). 
Table 3: Pairwise t-test on difference of total soil carbon between zones A, B, C and $\mathrm{H}$ in topsoil and subsoil layers under Vitellaria paradoxa and Parkia biglobosa at Tougouri (with the lowest rainfall level studied) in Burkina Faso (West Africa).

\begin{tabular}{|c|c|c|c|c|c|c|c|}
\hline Parameter & Tree species & Sites & Layers & $\begin{array}{c}\text { Compared } \\
\text { zones }\end{array}$ & Frequency & T-values & $\begin{array}{c}P \text { - } \\
\text { value } \\
\text { S }\end{array}$ \\
\hline \multirow{24}{*}{$\begin{array}{c}\text { Total } \\
\text { carbon }\end{array}$} & \multirow[t]{24}{*}{$V \cdot$ paradoxa } & \multirow[t]{24}{*}{ Tougouri } & \multirow[t]{6}{*}{010} & A-B & 8 & 0,002 & 0,999 \\
\hline & & & & $\mathrm{A}-\mathrm{C}$ & 8 & $-0,127$ & 0,902 \\
\hline & & & & A-H & 8 & 0,68 & 0,518 \\
\hline & & & & B-C & 8 & $-0,097$ & 0,926 \\
\hline & & & & B-H & 8 & 1,211 & 0,265 \\
\hline & & & & $\mathrm{C}-\mathrm{H}$ & 8 & 0,869 & 0,414 \\
\hline & & & \multirow[t]{6}{*}{1020} & A-B & 8 & 3,213 & 0,015 \\
\hline & & & & $\mathrm{A}-\mathrm{C}$ & 8 & 1,135 & 0,294 \\
\hline & & & & A-H & 8 & 1,472 & 0,185 \\
\hline & & & & B-C & 8 & $-1,4$ & 0,204 \\
\hline & & & & B-H & 8 & 0,483 & 0,644 \\
\hline & & & & $\mathrm{C}-\mathrm{H}$ & 8 & 1,177 & 0,278 \\
\hline & & & \multirow[t]{6}{*}{2030} & A-B & 8 & 2,318 & 0,054 \\
\hline & & & & $\mathrm{A}-\mathrm{C}$ & 8 & 2,778 & $\mathbf{0 , 0 2 7}$ \\
\hline & & & & $\mathrm{A}-\mathrm{H}$ & 8 & 0,861 & 0,418 \\
\hline & & & & $\mathrm{B}-\mathrm{C}$ & 8 & 0,903 & 0,396 \\
\hline & & & & B-H & 8 & $-0,217$ & 0,834 \\
\hline & & & & $\mathrm{C}-\mathrm{H}$ & 8 & $-0,916$ & 0,39 \\
\hline & & & \multirow[t]{6}{*}{3040} & A-B & 8 & $-0,351$ & 0,736 \\
\hline & & & & $\mathrm{A}-\mathrm{C}$ & 8 & 3,159 & 0,016 \\
\hline & & & & A-H & 8 & 3,369 & 0,012 \\
\hline & & & & B-C & 8 & 1,701 & 0,133 \\
\hline & & & & B-H & 8 & 2,361 & 0,05 \\
\hline & & & & C-H & 8 & 1,572 & 0,16 \\
\hline \multirow[t]{14}{*}{$\begin{array}{c}\text { Total } \\
\text { carbon }\end{array}$} & \multirow[t]{14}{*}{ P. biglobosa } & \multirow[t]{14}{*}{ Tougouri } & \multirow[t]{6}{*}{010} & A-B & 8 & $-0,341$ & 0,743 \\
\hline & & & & $\mathrm{A}-\mathrm{C}$ & 8 & 0,141 & 0,892 \\
\hline & & & & $\mathrm{A}-\mathrm{H}$ & 8 & 1,032 & 0,336 \\
\hline & & & & $\mathrm{B}-\mathrm{C}$ & 8 & 1,125 & 0,298 \\
\hline & & & & B-H & 8 & 1,958 & 0,091 \\
\hline & & & & $\mathrm{C}-\mathrm{H}$ & 8 & 1,863 & 0,105 \\
\hline & & & \multirow[t]{6}{*}{1020} & A-B & 8 & $-2,25$ & 0,059 \\
\hline & & & & A-C & 8 & $-2,463$ & 0,043 \\
\hline & & & & $\mathrm{A}-\mathrm{H}$ & 8 & 0,725 & 0,492 \\
\hline & & & & B-C & 8 & $-1,753$ & 0,123 \\
\hline & & & & B-H & 8 & 3,293 & $\mathbf{0 , 0 1 3}$ \\
\hline & & & & C-H & 8 & 3,554 & 0,009 \\
\hline & & & \multirow[t]{2}{*}{2030} & A-B & 8 & $-1,917$ & 0,097 \\
\hline & & & & A-C & 8 & 1,261 & 0,248 \\
\hline
\end{tabular}




\begin{tabular}{ccccc} 
& A-H & 8 & 0,536 & 0,609 \\
\cline { 2 - 5 } & B-C & 8 & 2,077 & 0,076 \\
\cline { 2 - 5 } & B-H & 8 & 2,138 & 0,07 \\
\cline { 2 - 5 } 3040 & C-H & 8 & $-0,241$ & 0,817 \\
\cline { 2 - 5 } & A-B & 8 & $-0,248$ & 0,811 \\
\cline { 2 - 5 } & A-C & 8 & 2,105 & 0,073 \\
\cline { 2 - 5 } & A-H & 8 & 0,714 & 0,498 \\
\hline & B-C & 8 & 1,015 & 0,344 \\
\hline B-H & 8 & 1,01 & 0,346 \\
\hline C-H & 8 & $-0,5$ & 0,632 \\
\hline
\end{tabular}

Significant $=\mathrm{P}<0.05$; Very significant $=\mathrm{P}<0.01 ;$ Highly significant $=\mathrm{P}<0.001$.
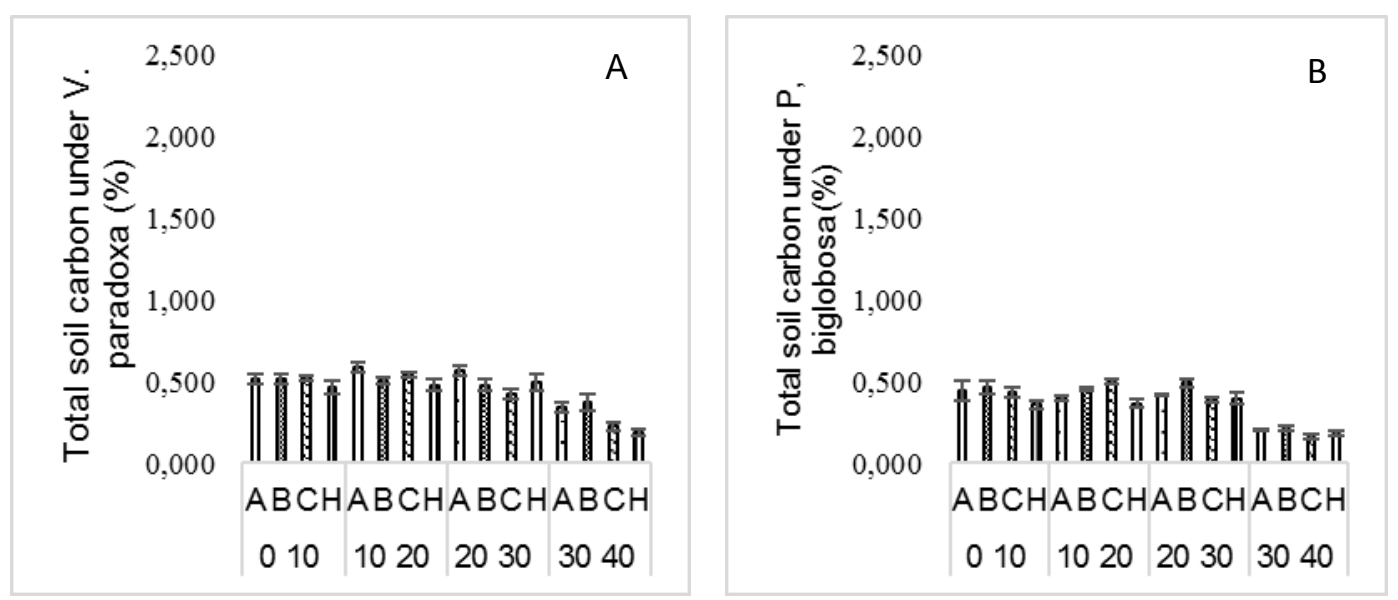

Figure 6: Total soil carbon variation between zones $\mathrm{A}, \mathrm{B}, \mathrm{C}$ and $\mathrm{H}$ in topsoil and subsoil layers under V. paradoxa $(\mathbf{A})$ and P. biglobosa $(\mathbf{B})$ at Tougouri (with the lowest rainfall level studied) in agroforestry parklands systems in Burkina Faso (West Africa).

Table 4: Pairwise t-test on difference of total soil carbon between zones A, B, C and $\mathrm{H}$ in topsoil and subsoil layers under Vitellaria paradoxa and Parkia biglobosa at Nobere (with the middle rainfall level studied) in Burkina Faso (West Africa).

\begin{tabular}{|c|c|c|c|c|c|c|c|}
\hline Parameter & $\begin{array}{c}\text { Tree } \\
\text { species }\end{array}$ & Site & Layers & $\begin{array}{c}\text { Compared } \\
\text { zones }\end{array}$ & Frequency & T-values & $\begin{array}{c}\text { P- } \\
\text { values }\end{array}$ \\
\hline \multirow[t]{8}{*}{$\begin{array}{c}\text { Total } \\
\text { carbon }\end{array}$} & $V \cdot$ paradoxa & Nobere & 010 & A-B & 8 & 0,432 & 0,679 \\
\hline & & & & $\mathrm{A}-\mathrm{C}$ & 8 & 1,362 & 0,215 \\
\hline & & & & A-H & 8 & 0,701 & 0,506 \\
\hline & & & & B-C & 8 & 0,933 & 0,382 \\
\hline & & & & B-H & 8 & 0,271 & 0,794 \\
\hline & & & & $\mathrm{C}-\mathrm{H}$ & 8 & $-0,712$ & 0,5 \\
\hline & & & 1020 & A-B & 8 & 0,473 & 0,651 \\
\hline & & & & $\mathrm{A}-\mathrm{C}$ & 8 & 2,456 & 0,044 \\
\hline
\end{tabular}


Y. N. COULIBALY et al. / Int. J. Biol. Chem. Sci. 14(9): 3030-3046, 2020

\begin{tabular}{|c|c|c|c|c|c|c|}
\hline & & & A-H & 8 & $-0,908$ & 0,394 \\
\hline & & & B-C & 8 & 1,767 & 0,121 \\
\hline & & & B-H & 8 & $-1,368$ & 0,214 \\
\hline & & & C-H & 8 & $-2,171$ & 0,066 \\
\hline & & 2030 & A-B & 8 & 0,969 & 0,365 \\
\hline & & & A-C & 8 & 1,662 & 0,141 \\
\hline & & & A-H & 8 & $-0,989$ & 0,356 \\
\hline & & & B-C & 8 & 0,646 & 0,539 \\
\hline & & & B-H & 8 & $-1,57$ & 0,16 \\
\hline & & & C-H & 8 & $-1,665$ & 0,14 \\
\hline & & 3040 & A-B & 8 & 1,432 & 0,195 \\
\hline & & & A-C & 8 & 1,695 & 0,134 \\
\hline & & & A-H & 8 & 0,126 & 0,903 \\
\hline & & & B-C & 8 & 0,689 & 0,513 \\
\hline & & & B-H & 8 & $-1,026$ & 0,339 \\
\hline & & & C-H & 8 & $-2,091$ & 0,075 \\
\hline \multirow[t]{24}{*}{$\begin{array}{c}P . \\
\text { biglobosa }\end{array}$} & \multirow[t]{24}{*}{ Nobere } & 010 & A-B & 8 & 1,008 & 0,347 \\
\hline & & & A-C & 8 & 3,013 & $\mathbf{0 , 0 2}$ \\
\hline & & & A-H & 8 & 2,174 & 0,066 \\
\hline & & & B-C & 8 & 2,465 & 0,043 \\
\hline & & & B-H & 8 & 1,485 & 0,181 \\
\hline & & & C-H & 8 & $-0,245$ & 0,814 \\
\hline & & 1020 & A-B & 8 & 2,919 & 0,022 \\
\hline & & & $\mathrm{A}-\mathrm{C}$ & 8 & 2,707 & $\mathbf{0 , 0 3}$ \\
\hline & & & A-H & 8 & 2,309 & 0,054 \\
\hline & & & B-C & 8 & 1,737 & 0,126 \\
\hline & & & B-H & 8 & 0,676 & 0,521 \\
\hline & & & C-H & 8 & $-1,779$ & 0,118 \\
\hline & & 2030 & A-B & 8 & 1,139 & 0,292 \\
\hline & & & A-C & 8 & 1,084 & 0,314 \\
\hline & & & A-H & 8 & 1,322 & 0,228 \\
\hline & & & B-C & 8 & 0,85 & 0,423 \\
\hline & & & B-H & 8 & 0,964 & 0,367 \\
\hline & & & C-H & 8 & $-0,263$ & 0,8 \\
\hline & & 3040 & A-B & 8 & 1,385 & 0,209 \\
\hline & & & A-C & 8 & 1,61 & 0,151 \\
\hline & & & A-H & 8 & 1,534 & 0,169 \\
\hline & & & B-C & 8 & 1,271 & 0,244 \\
\hline & & & B-H & 8 & 0,8 & 0,45 \\
\hline & & & C-H & 8 & $-0,143$ & 0,89 \\
\hline
\end{tabular}

Significant $=\mathrm{P}<0.05 ;$ Very significant $=\mathrm{P}<0.01 ;$ Highly significant $=\mathrm{P}<0.001$. 

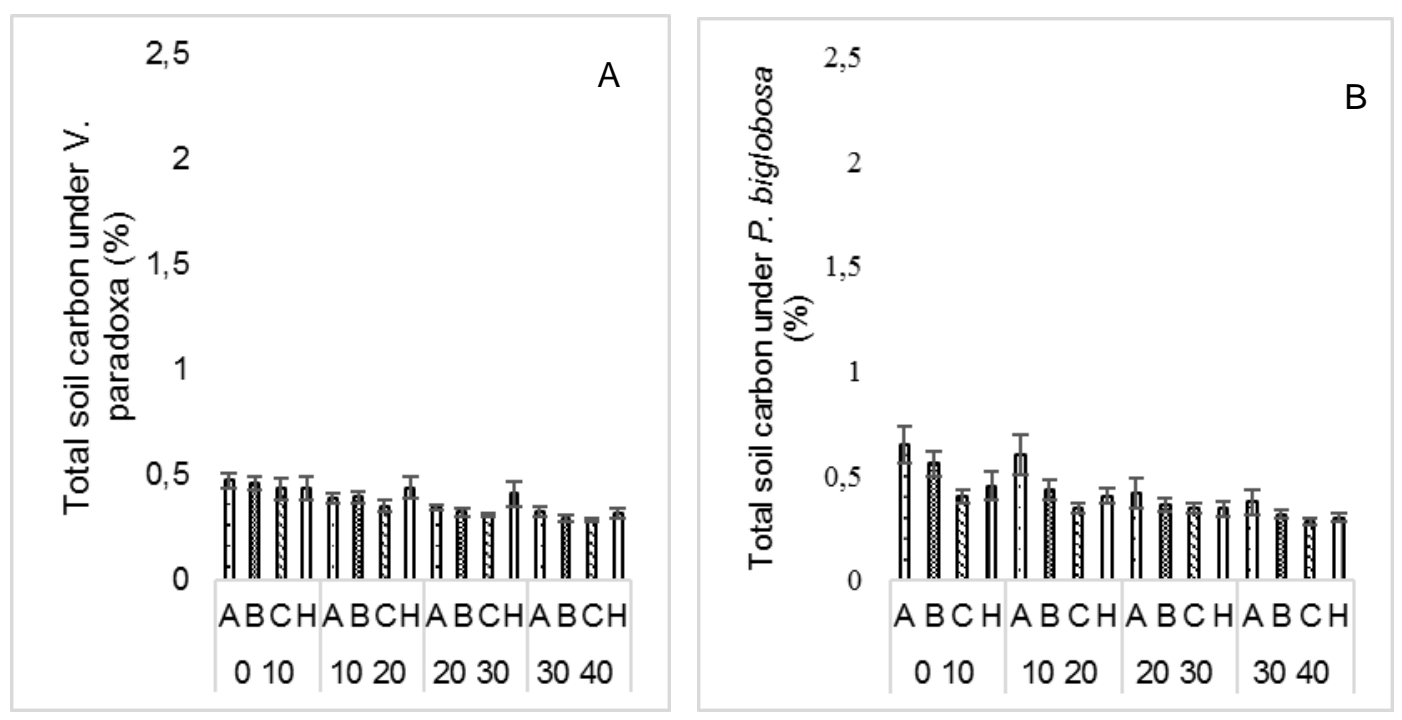

Figure 7: Total soil carbon variation between zones A, B, C and $\mathrm{H}$ in topsoil and subsoil layers under V. paradoxa (A) and P. biglobosa (B) at Nobere (with the middle rainfall level studied) in agroforestry parklands systems in Burkina Faso (West Africa).

Table 5: Pairwise t-test on difference of total soil carbon between zones A, B, C and H in topsoil and subsoil layers under Vitellaria paradoxa and Parkia biglobosa at Sokouraba (with the highest rainfall level studied) in Burkina Faso (West Africa).

\begin{tabular}{|c|c|c|c|c|c|c|c|}
\hline Parameter & $\begin{array}{c}\text { Tree } \\
\text { species }\end{array}$ & Site & Layers & $\begin{array}{c}\text { Compared } \\
\text { zones }\end{array}$ & Frequency & T-values & $\begin{array}{c}\mathbf{P}- \\
\text { values }\end{array}$ \\
\hline \multirow{21}{*}{$\begin{array}{c}\text { Total } \\
\text { carbon }\end{array}$} & \multirow[t]{21}{*}{$V$. paradoxa } & \multirow[t]{21}{*}{ Sokouraba } & \multirow[t]{6}{*}{010} & A-B & 8 & 2,339 & 0,052 \\
\hline & & & & A-C & 8 & 2,737 & $\mathbf{0 , 0 2 9}$ \\
\hline & & & & A-H & 8 & 2,879 & 0,024 \\
\hline & & & & B-C & 8 & 0,942 & 0,378 \\
\hline & & & & B-H & 8 & 1,146 & 0,29 \\
\hline & & & & C-H & 8 & 0,356 & 0,732 \\
\hline & & & \multirow[t]{6}{*}{1020} & A-B & 8 & 0,976 & 0,362 \\
\hline & & & & A-C & 8 & 3,289 & $\mathbf{0 , 0 1 3}$ \\
\hline & & & & A-H & 8 & 1,954 & 0,092 \\
\hline & & & & B-C & 8 & 0,66 & 0,531 \\
\hline & & & & B-H & 8 & 0,629 & 0,549 \\
\hline & & & & C-H & 8 & 0,228 & 0,826 \\
\hline & & & \multirow[t]{6}{*}{2030} & A-B & 8 & 1,33 & 0,225 \\
\hline & & & & $\mathrm{A}-\mathrm{C}$ & 8 & 1,262 & 0,247 \\
\hline & & & & A-H & 8 & 0,959 & 0,369 \\
\hline & & & & B-C & 8 & 0,548 & 0,601 \\
\hline & & & & B-H & 8 & $-0,249$ & 0,81 \\
\hline & & & & $\mathrm{C}-\mathrm{H}$ & 8 & $-0,714$ & 0,498 \\
\hline & & & \multirow[t]{3}{*}{3040} & A-B & 8 & 1,546 & 0,166 \\
\hline & & & & A-C & 8 & 2,695 & $\mathbf{0 , 0 3 1}$ \\
\hline & & & & A-H & 8 & 0,033 & 0,974 \\
\hline
\end{tabular}




\begin{tabular}{|c|c|c|c|c|c|c|}
\hline & & & \multirow{3}{*}{$\frac{\text { B-C }}{\frac{B-H}{C-H}}$} & \multirow{2}{*}{$\begin{array}{l}8 \\
8\end{array}$} & \multirow{2}{*}{$\begin{array}{c}0,431 \\
-2,51\end{array}$} & \multirow{2}{*}{$\begin{array}{l}0,68 \\
\mathbf{0 , 0 4}\end{array}$} \\
\hline & & & & & & \\
\hline & & & & 8 & $-2,344$ & 0,052 \\
\hline \multirow{24}{*}{$\begin{array}{c}P . \\
\text { biglobosa }\end{array}$} & \multirow[t]{24}{*}{ Sokouraba } & \multirow[t]{6}{*}{010} & A-B & 8 & 0,404 & 0,698 \\
\hline & & & $\mathrm{A}-\mathrm{C}$ & 8 & 3,803 & $\mathbf{0 , 0 0 7}$ \\
\hline & & & A-H & 8 & 3,349 & 0,012 \\
\hline & & & B-C & 8 & 1,305 & 0,233 \\
\hline & & & B-H & 8 & 1,706 & 0,132 \\
\hline & & & $\mathrm{C}-\mathrm{H}$ & 8 & 1,552 & 0,165 \\
\hline & & \multirow[t]{6}{*}{1020} & A-B & 8 & 3,303 & 0,013 \\
\hline & & & $\mathrm{A}-\mathrm{C}$ & 8 & 4,025 & 0,005 \\
\hline & & & A-H & 8 & 4,017 & 0,005 \\
\hline & & & B-C & 8 & 1,748 & 0,124 \\
\hline & & & B-H & 8 & 2,591 & 0,036 \\
\hline & & & $\mathrm{C}-\mathrm{H}$ & 8 & 0,029 & 0,978 \\
\hline & & \multirow[t]{6}{*}{2030} & $A-B$ & 8 & 0,459 & 0,66 \\
\hline & & & $\mathrm{A}-\mathrm{C}$ & 8 & 2,302 & 0,055 \\
\hline & & & A-H & 8 & 3,467 & 0,01 \\
\hline & & & B-C & 8 & 1,272 & 0,244 \\
\hline & & & B-H & 8 & 1,993 & 0,087 \\
\hline & & & $\mathrm{C}-\mathrm{H}$ & 8 & 0,243 & 0,815 \\
\hline & & \multirow[t]{6}{*}{3040} & A-B & 8 & 1,156 & 0,285 \\
\hline & & & $\mathrm{A}-\mathrm{C}$ & 8 & 2,938 & 0,022 \\
\hline & & & A-H & 8 & 1,037 & 0,334 \\
\hline & & & B-C & 8 & 1,04 & 0,333 \\
\hline & & & B-H & 8 & 0,447 & 0,668 \\
\hline & & & C-H & 8 & $-0,099$ & 0,924 \\
\hline
\end{tabular}
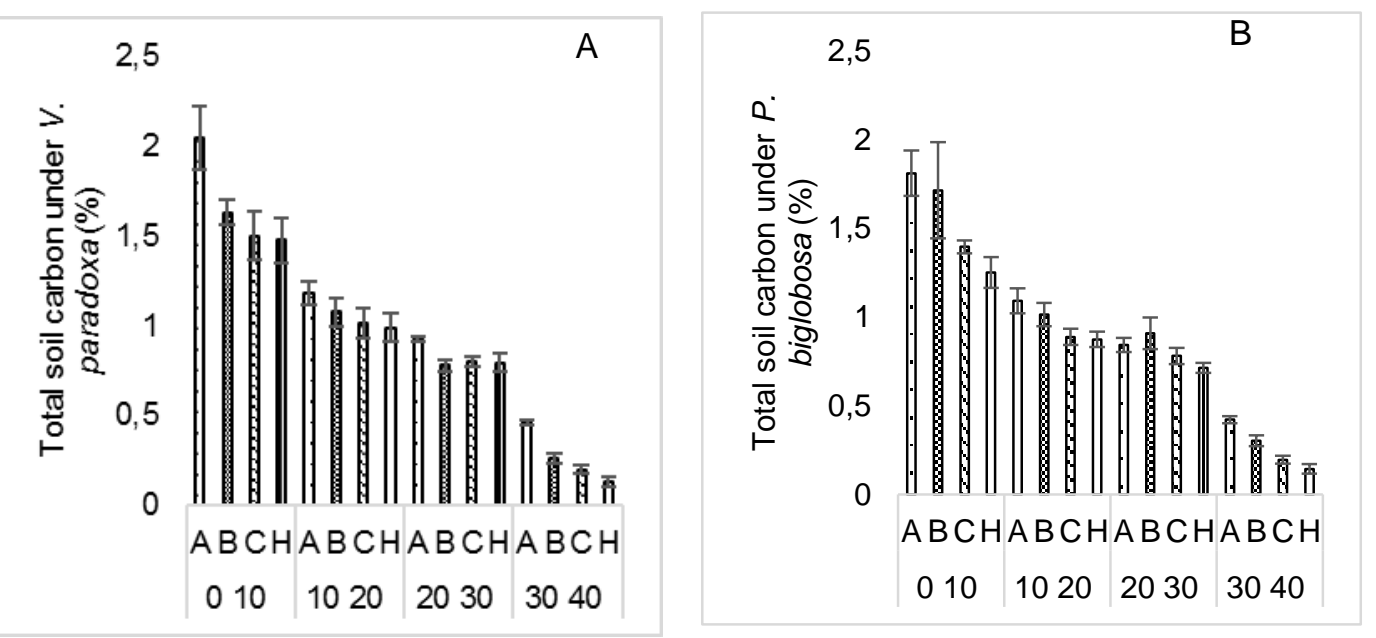

Figure 8: Total soil carbon variation between zones $\mathrm{A}, \mathrm{B}, \mathrm{C}$ and $\mathrm{H}$ in topsoil and subsoil layers under V. paradoxa (A) and P. biglobosa (B) at Sokouraba (with the highest rainfall level studied) in agroforestry parklands systems in Burkina Faso (West Africa). 
Table 6: The magnitude of the effects of $V$. paradoxa and $P$. biglobosa on total soil carbon at Tougouri (with the lowest rainfall level studied), Nobere (with the middle rainfall level studied) and Sokouraba (with the highest rainfall level studied) in Burkina Faso (West Africa).

\begin{tabular}{llccc}
\hline Sites & Tree species & Under trees & Control plot & Difference \\
\hline \multirow{2}{*}{ Tougouri } & V. paradoxa & 0,467 & 0,408 & 0,060 \\
& P. biglobosa & 0,382 & 0,326 & 0,056 \\
\hline \multirow{2}{*}{ Nobere } & V. paradoxa & 0,362 & 0,403 & $-0,041$ \\
& P. biglobosa & 0,424 & 0,370 & 0,055 \\
\hline \multirow{2}{*}{ Sokouraba } & V. paradoxa & 1,116 & 1,033 & 0,083 \\
& P. biglobosa & 1,093 & 0,916 & 0,178 \\
\hline
\end{tabular}

\section{DISCUSSION}

The effects of agroforestry parklands on soil organic carbon depend on biophysical and socioeconomic characteristics of the system (Nair et al., 2009; Nair and Nair, 2014). Total soil carbon was higher at Sokouraba with the highest rainfall level studied and it was not significantly different between $V$. paradoxa and P. biglobosa for topsoil and subsoil layers probably due to better climatic conditions and land management practices (Martin et al., 2010; Mathayo et al., 2016). The lowest population increase, population density and livestock development at Sokouraba could permit shorter period of land utilization with the possibility to still practice fallows allowing reconstitution of soil organic carbon stock. The importance of the precipitation and consequently improved soil humidity content at Sokouraba could also explained highest values of its total soil carbon due to the acceleration of decomposition of organic matter as reported by Manns and Berg (2014), Klopfenstein et al. (2015) and Mathayo et al. (2016). Higher total soil carbon observed at Sokouraba could also probably due to higher tree aboveground biomass inputs in soil. Tougouri with the lowest rainfall level studied and Nobere with the middle rainfall level studied characterised by high population, population density and livestock development coupled with low precipitation could explain their lowest total soil carbon content due to the degradation of the system. Dryness of the upper soil forces the fine roots to develop deeper than the layers we have sampled, wind blowing away the litter, heat accelerating the decomposition of the above and below-ground organic inputs and finally livestock transferring the litter it consumes could explain the lowest total soil carbon content at Tougouri and Nobere. At Tougouri and Nobere, the anthropogenic effects on trees degradation due to the use of non-timber forest products for food, wood energy, medicines and livestock development could reduce trees performance to provide environmental services such as soil fertility improvement in parklands systems through a low soil organic carbon reconstitution in soil due to a low tree aboveground biomass inputs in soil. Aboveground biomass plays an important role in the soil organic carbon through litter fall and combination of climate, soil types and management practices (Mathayo et al., 2016). Coulibaly et al. (2014) reported in their study about simulation of precipitation increase effect on tree aboveground biomass, an increase of tree aboveground biomass when precipitation increases and inversely. Highest values of total soil carbon at Sokouraba could give soils better physical and chemical properties improving crop performance. The presence of important quantity of total soil carbon reduces soil erosion due to the structural stability of soil improved (Garcia-Barrios and Ong, 2004). Improving soil structural stability leads to a better circulation of water and air in the 
soil (Munoz et al., 2007) and then increases the nutrients availability for tree and crop roots (Brussaard et al., 2007). Coulibaly et al. (2014) reported higher sorghum bicolor (L) Moench biomass and yield at Sokouraba compared to Nobere and Tougouri. It could be suggested to Tougouri and Nobere farmers the supply of organic matter or crop rotation for improving soil carbon and crop productivity (Raffa et al., 2015).

At Tougouri, total soil carbon at topsoil $10-20 \mathrm{~cm}$ and subsoil $30-40 \mathrm{~cm}$ and magnitude of total soil carbon improvement was higher under $V$. paradoxa probably due to its open canopy structure (Bazié et al., 2012) which allows more water at this site with low precipitation reaching the soil (Zomboudre et al., 2005) increasing then soil humidity leading to better litter and crop roots decomposition process. However, at Nobere, total soil carbon at topsoil $10-20 \mathrm{~cm}$ and magnitude of total soil carbon improvement was higher under $P$. biglobosa. The magnitude of total soil carbon improvement was also higher under $P$. biglobosa at Sokouraba. These results corroborate Atchada et al. (2019) who reported higher soil organic carbon in topsoil layer $0-10 \mathrm{~cm}$. These were probably due to decomposition of more tree roots in addition to others litter with rainfall increase because it has been reported that $P$. biglobosa roots are important in surface (Bayala et al., 2004). The positive magnitude of $V$. paradoxa and P. biglobosa on total soil carbon showed that under both tree species it has been increased compared to control plot at all the study sites corroborating several authors who reported positive effects of agroforestry practices on soil organic carbon (Lorenz et al., 2014; Mathayo et al., 2016; Sileshi, 2016; Aryal et al., 2019; Bayala et al., 2019). Total soil carbon was influenced by tree species along a rainfall gradient suggesting promotion of $V$. paradoxa in agroforestry parklands for soil fertility and crop productivity improvement, particularly in areas with low precipitation.

The decrease trend of total soil carbon under both tree species at topsoil and subsoil layers from zone $\mathrm{A}$ at all the study sites corroborates several authors who reported positive effects of tree on soil organic carbon in zone under tree near trunk in agroforestry parklands (Takimoto et al., 2009; Bambrick et al., 2010; Howlett et al., 2011) probably due to higher biomass inputs, slower decomposition and reduction of frequent tillage (a couple of times during the growing season). Tillage using animal traction is done very close to trunks of parklands trees where tree density is low, and this possibly accelerates the decomposition of organic matter in surface soil, and thus reduces soil organic carbon accumulation (Takimoto et al., 2009). Higher total soil carbon at topsoil layers compared to subsoil layers in zones under trees for all sites under both tree species could suggest that major soil organic carbon established in agroforestry parklands derives from the slow decomposition process of tree leaves from aboveground biomass (Bayala et al., 2006). It could be suggested to farmers to reduce tillage in agroforestry parklands to enhance potential of trees in improving total soil carbon and crop productivity.

\section{Conclusion}

Investigating trees effects on total soil carbon in agroforestry parklands along a rainfall gradient is crucial as it gives opportunities for recommendations of tree management options which increase soil carbon and crop productivity for parklands adaptation to climate change. Total soil carbon was lower when rainfall decreased and it was improved under tree compared to control plot whatever the rainfall level. Total soil carbon varied according to tree species along a rainfall gradient. For topsoil and subsoil layers, total soil carbon was higher under $V$. paradoxa at Tougouri. It was higher under $P$. biglobosa and $V$. paradoxa at topsoil and subsoil layers when rainfall slightly increased. The higher magnitudes were observed at Sokouraba for P. biglobosa and V. paradoxa. For Tougouri, the magnitude was higher under $V$. paradoxa while at Nobere and Sokouraba it was higher under P. biglobosa. Total soil carbon decreased from zone A to control plot and from the topsoil to subsoil 
layers at all rainfall levels under both tree species. It could be concluded that trees improve total soil carbon, but the importance of their contributions in improving total soil carbon depends on tree species according to the rainfall level, suggesting an appropriate choice of tree species according to a specific climatic condition for parklands adaptation to climate change. This research could suggest farmers, and particularly those in areas with low rainfall, the promotion of $V$. paradoxa in agroforestry parklands through natural regeneration and selective weeding improving soil carbon and crop productivity. However, future research should aim at measuring both total carbon and organic carbon to be able to disentangle the contribution from trees from that of carbon from soil parent materials (inorganic source).

\section{COMPETING INTERESTS}

The authors declare that they have no competing interests.

\section{AUTHORS' CONTRIBUTIONS}

YNC is the first author of this article. He developed the research protocol, collected and analysed the data and initiated this paper in the framework of his PhD research. JB and GZ supervised the work of the $\mathrm{PhD}$ studies and they have contributed in revising this paper. TG contributed in data analysis, revising and editing english language of the manuscript.

\section{ACKNOWLEDGMENTS}

We would like to thank farmers of Tougouri, Nobere and Sokouraba for their valuable contribution in data collection and their attention to the experiments throughout. We are also grateful to the technicians for their assistance in the data measurements. Thank you to my supervisors for their guidance and advice.

\section{REFERENCES}

Abdou MM, Alzouma ZM, Kadri A, Ambouta JMK, Dan Lamso N. 2013. Effet de l'arbre Acacia senegal sur la fertilité des sols de gommeraies au Niger. Int. J. Biol.
Chem. Sci., 7(6): 2328-2337. DOI: 10.4314/ijbcs.v7i6.13

Aliou SB, Koné I, Gnangle B, Césaire AN. 2013. Effet d'un système agroforestier à karité (Vitellaria paradoxa C. F Gaertn) sur le sol et le potentiel de production du mais (Zea mays L.) en zone Soudanienne du Bénin. Int. J. Biol. Chem. Sci., 6(5): 2066-2082.

DOI: http://dx.doi.org/10.4314/ijbcs.v6i5.16

Aryal DR, Gómez-González RR, HernándezNuriasmú R, Morales-Ruiz DE. 2019. Carbon stocks and tree diversity in scattered tree silvopastoral systems in Chiapas, Mexico. Agroforest. Syst., 93: 213-227.

DOI:

https://doi.org/10.1007/s10457-0180310-y

Atchada C, Zoffoun A, Akplo T, Azontonde A, Tente A, Djego J. 2019. Modes d'utilisation des terres et stock de carbone organique du sol dans le bassin supérieur de Magou au Bénin. Int. J. Biol. Chem. Sci., 12(6): 2818-2829. DOI: https://dx.doi.org/10.4314/ijbcs.v12i6.27

Bambrick AD, Whalen JK, Bradley RL, Cogliastro A, Gordon AM, Olivier A, Thevathasan NV. 2010. Spatial heterogeneity of soil organic carbon in tree-based intercropping systems in Quebec and Ontario, Canada. Agroforest. Syst., 79: 343-353. DOI: 10.1007/s10457-010-9305-z

Bationo A, Kihara J, Vanlauwe B, Waswa B, Kimetu J. 2007. Soil organic carbon dynamics, functions and management in West African agro-ecosystems. Agric. Syst. J., $\quad \mathbf{9 4}$ : 13-25. DOI: $10.1016 /$ j.agsy.2005.08.011

Bayala J, Teklehaimanot Z, Ouédraogo SJ. 2004. Fine root distribution of pruned trees and associated crops in parkland system in Burkina Faso. Agroforest. Syst., $\quad 60$ : 13- 26. DOI:10.1023/B:AGFO.0000009401.963 09.12

Bayala J, Balesdent J, Marol C, Zapata F, Teklehaimanot Z, Ouedraogo SJ. 2006. Relative contribution of trees and crops to soil carbon content in a parkland 
system in Burkina Faso using variations in natural 13C abundance. Nutrient Cycle. Agroecosyst., 76: 193-201. DOI: https://doi.org/10.1007/s10705-0051547-1

Bayala J, Sanou J, Bazié HR, Coe R, Kalinganire A, Sinclair FL. 2019. Regenerated trees in farmers' fields increase soil carbon across the Sahel. Agroforest. Syst., 94: 401-415. DOI: https://doi.org/10.1007/s10457-01900403-60123456789

Bazie HR, Bayala J, Zombre G, Sanou J, Ilstedt U. 2012. Separating competitionrelated factors limiting crop performance in an agroforestry parkland system in Burkina Faso. Agroforest. Syst., 84:377388. DOI: 10.1007/s10457-012-9483-y

Brussaard L, Pulleman MM, Ouédraogo E, Mando A, Six J. 2007. Soil fauna and soil function in the fabric of the food web. Pedobiologia, 50: 447-462. DOI: 10.1016/j.pedobi.2006.10.007

Coulibaly YN, Mulia R, Sanou J, Zombré G, Bayala J, Kalinganire A, van Noordwijk M. 2014. Crop production under different rainfall and management conditions in agroforestry parkland systems in Burkina Faso: observations and simulation with WaNuLCAS model. Agroforest. Syst., 88: 13-28. DOI: 10.1007/s10457-013-9651-8

Direction Générale de la Météorologie du Burkina Faso. 2013. Base de données climatiques du Burkina Faso. DGMBF, Ouagadougou, Burkina Faso.

Du C, Zhou J. 2009. Evaluation of soil fertility using infrared spectroscopy: a review. Environmental Chemistry Letter, 7: 97-113.

DOI: https://doi.org/10.1007/s10311-0080166-x

Garcia-Barrios I, Ong CK. 2004. Ecological interaction lessons and design tools in tropical agroforestry systems. Agroforest. Syst., 61: 221-236. DOI: https://doi.org/10.1023/B:AGFO.000002 9001.81701.f0

Howlett DS, Moreno G, Mosquera Losada MR, Nair PKR, Nair VD. 2011. Soil carbon storage as influenced by tree cover in the Dehesa cork oak silvopasture of central western Spain. $J$. of Environ. Monit., 13: 1897-1904. DOI: https://doi.org/10.1039/C1EM10059A

Institut National de la Statistique et de la Démographie. 2018. Annuaire statistique 2017. INSD, 383p

Klopfenstein ST, Hirmas DR, Johnson WC. 2015. Relationships between soil organic carbon and precipitation along a climosequence in loess-derived soils of the Central Great Plains, USA. $J$. Catena., 133: 25-34. DOI: $10.1016 /$ j.catena.2015.04.015

Lorenz K, Lal R. 2014. Soil organic carbon sequestration in agroforestry systems. A review. Agron. Sustain. Dev., 34: 443454. DOI: $10.1007 / \mathrm{s} 13593-014-0212-y$

Manns HR, Berg AA. 2014. Importance of soil organic carbon on surface soil water content variability among agricultural fields. J. Hydrol., 516: 297-303. DOI: $10.1016 /$ j.jhydrol.2013.11.018

Martin D, Lal T, Sachdev CB, Sharma JP. 2010. Soil organic carbon storage changes with climate change, landform and land use conditions in Garhwal hills of the Indian himalayan mountains. Agric. Ecosyst. Environ., 138: 64-73. DOI: $10.1016 /$ j.agee.2010.04.001

Mathayo MM, Majule AE, Sinclair F, Marchant R. 2016. Relationships between on-farm tree stocks and soil organic carbon along an altitudinal gradient, Mount Kilimanjaro, Tanzania. Trees Livelihoods, 25(4): 255-266. DOI: https://doi.org/10.1080/14728028.2016.1 202790

Munoz C, Zagal E, Ovalle C. 2007. Influence of trees on soil organic matter in Mediterranean agroforestry systems: an example from the Espinal of central Chile. Europ. J. Soil Sci., 58: 728-735. DOI: $\quad$ https://doi.org/10.1111/j.13652389.2006.00858.x

Nair PKR, Kumar BM, Nair VD. 2009. Agroforestry as a strategy for carbon sequestration. J. Plant. Nutr. Soil Sci., 
172: 10-23. DOI : $10.1002 /$ jpln.200800030

Nair PKR, Nair VD. 2014. Solid-fluid-gas : the state of knowledge on carbonsequestration potential of agroforestry systems in Africa. Curr. Opin. Environ. Sustain., 6: 22-27. DOI: https://doi.org/10.1016/j.cosust.2013.07. 014

Ndiaye SSA, Elhadji F, Tala G, Hank M, Camire C. 2012. Cordyla pinnata améliore les propriétés du sol et la productivité des cultures. Int. J. Biol. Chem. Sci., 6(2): 714-725. DOI: http://dx.doi.org/10.4314/ijbcs.v6i2.15

Raffa DW, Bogdanski A, Tittonell P. 2015. How does crop residue removal affect soil organic carbon and yield? A hierarchical analysis of management and environmental factors. Biomass Bioenergy, 81: 345-355. DOI: 10.1016/j.biombioe.2015.07.022

Shepherd KD, Walsh MG. 2002. Development of reflectance spectral libraries for characterization of soil properties. Soil Sci. Society of America J., 66: $\quad 988-998 . \quad$ DOI: 10.2136/sssaj2002.0988

Sileshi GW. 2016. The magnitude and spatial extent of influence of Faidherbia albida trees on soil properties and primary productivity in drylands. J. Arid. Environ., $\quad$ 132: $1-14$. DOI: $10.1016 /$ j.jaridenv.2016.03.002.

Takimoto A, Nair VD, Nair RPK. 2009. Contribution of trees to soil carbon sequestration under agroforestry systems in the West African Sahel. Agroforest. Syst., 76: 11-25. DOI: https://doi.org/10.1007/s10457-0089179-5

Traore K, Ganry F, Oliver R, Gigou J. 2004. Litter production and soil fertility in a Vitellaria paradoxa Parkland in a catena in southern Mali. Arid Land Res. Management, 18: 359- 368. DOI: https://doi.org/10.1080/15324980490497 393

Zomboudré G, Zombré G, Ouedraogo $\mathrm{M}$, Guinko S, Roy Macauley H. 2005. Réponse physiologique et productivité des cultures dans un système agroforestier traditionnel : cas du maïs (Zea mays L.) associé au karité (Vitellaria paradoxa Gaertn.) dans la zone est du Burkina Faso. BASE, 9: 7585 . 